\title{
BI-INVARIANT SCHWARTZ MULTIPLIERS AND LOCAL SOLVABILITY ON NILPOTENT LIE GROUPS
}

\author{
JOE W. JENKINS
}

Let $X$ denote a finite-dimensional vector space with a fixed positive definite inner product, and let $\mathscr{S}(X)$ denote the Schwartz space on $X$. We let $\mathscr{M} \mathscr{S}(X)$ denote the space of continuous endomorphisms of $\mathscr{S}(X)$ that commute with the action of $X$ on $\mathscr{S}(X)$. The elements of $\mathscr{M} \mathscr{S}(X)$ are given by convolution by tempered distributions; i.e., for $E \in \mathscr{M} \mathscr{S}(X)$ there is a $D_{E} \in \mathscr{S}^{*}(X)$ such that $E f(x)=\left\langle D_{E}, l_{x} \breve{f}\right\rangle:=D_{E} * f(x)$, where $f(x)=f(-x)$ and $l_{x} f(y)=f(y-x)$. Conversely, if $D \in \mathscr{S}^{*}(X)$, then one can easily see that $E_{D}: f \rightarrow D * f$ is a mapping of $\mathscr{S}(X)$ into the smooth functions on $X$ that commutes with translation. Schwartz $[\mathbf{S}]$ shows that $E_{D} \in \mathscr{M S}(X)$ if and only if $\hat{D}$, the Fourier transform of $D$, is given by a smooth function on $X^{*}$ which has polynomial bounds on all derivatives. In this note we announce analogues of these results for arbitrary nilpotent Lie groups. Complete proofs will appear elsewhere.

Let $N$ denote a connected, simply connected nilpotent Lie group, with Lie algebra $\mathfrak{n}$. The exponential mapping, exp: $\mathfrak{n} \rightarrow N$, is a diffeomorphism, and in terms of the corresponding coordinates left and right translation on $N$ are polynomial mappings. Thus, if $\mathscr{S}(N)$ denotes the image under composition with exp of $\mathscr{S}(\mathfrak{n})$, the right and left action of $N$ on $\mathscr{S}(N)$ are continuous endomorphisms, where $\mathscr{S}(N)$ is topologized so that composition with exp is an isomorphism from $\mathscr{S}(\mathfrak{n})$ to $\mathscr{S}(N)$. We denote by $\mathscr{S}^{*}(N)$ the dual of $\mathscr{S}(N)$, the space of tempered distributions on $N$.

For $f \in \mathscr{S}(N)$, the Fourier transform of $f, \hat{f}$, is defined on $\mathfrak{n}^{*}$, the dual of $\mathfrak{n}$, by

$$
\hat{f}(\xi)=\int_{\mathfrak{n}} f(\exp X) e^{-2 \pi i\langle\xi, X\rangle} d X .
$$

One has that $f \rightarrow \hat{f}$ is an isomorphism from $\mathscr{S}(N)$ onto $\mathscr{S}\left(\mathfrak{n}^{*}\right)$. For $D \in$ $\mathscr{S}^{*}(N), \widehat{D}$ is defined on $\mathscr{S}\left(\mathfrak{n}^{*}\right)$ by $\langle\widehat{D}, f\rangle=\langle D, \hat{f} \circ \log \rangle$, where log denotes the inverse of exp.

Let $\mathrm{Ad}^{*}$ denote the coadjoint representation of $N$ on $\mathfrak{n}^{*}$. A tempered distribution $D$ on $\mathfrak{n}^{*}$ is said to be $\operatorname{Ad}^{*}$-invariant if $\left\langle D, f \circ \operatorname{Ad}^{*} x\right\rangle=\langle D, f\rangle$ for all $x \in N$ and $f \in \mathscr{S}\left(\mathfrak{n}^{*}\right)$. A tempered distribution $D$ on $N$ is said to be bi-invariant if $\left\langle D, \mathfrak{r}_{x^{-1}} f\right\rangle=\left\langle D, l_{x} f\right\rangle$ for all $f \in \mathscr{S}(N)$, where $\mathfrak{r}_{x} f(y)=f(y x)$ and $l_{x} f(y)=f\left(x^{-1} y\right)$ for all $x, y \in N$. A straightforward computation shows that an element $D \in \mathscr{S}(N)$ is bi-invariant if and only if $\widehat{D}$ is $\mathrm{Ad}^{*}$-invariant.

Received by the editors January 22, 1988.

1980 Mathematics Subject Classification (1985 Revision). Primary 22E30, 43A55.

This research supported in part by a grant from the National Science Foundation. 
Let $\mathscr{M} \mathscr{S}(N)$ denote the space of continuous endomorphisms on $\mathscr{S}(N)$ that commute with both right and left translations by elements of $N$. As in the Euclidean case, one has that for each $E \in \mathscr{M} \mathscr{S}(N)$ there is a $D_{E} \in \mathscr{S}^{*}(N)$ such that $E f=D_{E} * f$, where, as before, $D_{E} * f(x):=\left\langle D_{E}, l_{x} \breve{f}\right\rangle$. If $D \in$ $\mathscr{S}^{*}(N)$ we denote by $E_{D}$ the mapping defined on $\mathscr{S}(N)$ by $E_{D} f=D * f$.

Let $P B_{N}^{\infty}\left(\mathfrak{n}^{*}\right)$ denote the space of smooth, $\mathrm{Ad}^{*}$-invariant functions defined on $\mathfrak{n}^{*}$ with polynomial bounds on all derivatives. This space is topologized using the seminorms $\nu_{i j}$ defined on $P B_{N}^{\infty}\left(\mathfrak{n}^{*}\right)$ by

$$
\nu_{i j}(\theta)=\sup _{|\alpha| \leq j} \sup _{\xi \in \mathfrak{n}^{*}}\left|\partial^{\alpha} \theta(\xi)\right| /\left(1+\|\xi\|^{2}\right)^{i}
$$

where $\partial^{\alpha}$ denotes the standard differential operator corresponding to the multi-index $\alpha$, and some fixed basis of $\mathfrak{n}^{*}$. A sequence $\left\{E_{n}\right\} \subset \mathscr{M} \mathscr{S}(N)$ converges to 0 if $E_{n} f \rightarrow 0$ in $\mathscr{S}(N)$ for each $f \in \mathscr{S}(N)$.

THEOREM A. The mapping $\mathscr{M} \mathscr{S}(N) \rightarrow P B_{N}^{\infty}\left(\mathfrak{n}^{*}\right): E \rightarrow \widehat{D}_{E}$ is a homeomorphism and an algebra isomorphism, the products being composition on $\mathscr{M S}(N)$ and pointwise multiplication on $P B_{N}^{\infty}\left(\mathfrak{n}^{*}\right)$.

For $\xi \in \mathfrak{n}^{*}$, let $\pi_{\xi}$ denote the irreducible unitary representation of $N$ that corresponds to the $\mathrm{Ad}^{*}$-orbit of $\xi$ by the Kirillov theory. For $\theta \in P B_{N}^{\infty}\left(\mathfrak{n}^{*}\right)$, let $D_{\theta}$ be the tempered distribution on $N$ with Fourier transform $\theta$.

THEOREM B. For $\theta \in P B_{N}^{\infty}\left(\mathfrak{n}^{*}\right), f \in \mathscr{S}(N)$, and $\xi \in \mathfrak{n}^{*}$,

$$
\pi_{\xi}\left(D_{\theta} * f\right)=\theta(\xi) \pi_{\xi}(f)
$$

As an application of these results, we consider the question of local solvability. Recall that a left invariant differential operator $L$ on $N$ is said to be locally solvable if there is an open set $U \subset N$ such that $C_{c}^{\infty}(U) \subset L\left(C^{\infty}(U)\right)$.

Let $o(\xi)$ denote the $\mathrm{Ad}^{*}$-orbit in $\mathfrak{n}^{*}$ that contains $\xi$, and having fixed a norm on $\mathfrak{n}^{*}$, set $|o(\xi)|=\inf \left\{\left\|\xi^{\prime}\right\|: \xi^{\prime} \in o(\xi)\right\}$. Suppose that $N$ contains a discrete, cocompact subgroup $\Gamma$. Then $L^{2}(\Gamma \backslash N)$ is a direct sum of subspaces $\mathscr{H}_{\xi}$ such that the restriction to $\mathscr{H}_{\xi}$ of right translation is a finite multiple of $\pi_{\xi}$. We denote by $(\Gamma \backslash N)_{0}^{\wedge}$ the elements of $\widehat{N}$ appearing in this decomposition that are in general position.

THEOREM C. Let $L$ be a left invariant differential operator on $N$. Suppose that for each $\pi_{\xi} \in(\Gamma \backslash N)_{0}^{\wedge}, \pi_{\xi}(L)$ has a bounded right inverse $A_{\xi}$ on $\mathscr{H}_{\xi}$, and that the norm of $A_{\xi}$ is bounded by a polynomial in $|o(\xi)|$. Then $L$ is locally solvable.

The proof of Theorem A requires the introduction of somewhat more general spaces. Let $h$ be a subspace of the center of $n$, and let $\lambda \in h^{*}$. We define the unitary character $\chi_{\lambda}$ on $H:=\exp (h)$ by $\chi_{\lambda}(\exp X)=e^{2 \pi i\langle\lambda, X\rangle}$, and denote by $\mathscr{S}\left(N / H, \chi_{\lambda}\right)$ the space of all smooth functions $f$ defined on $N$ such that $f(x y)=\chi_{\lambda}(y) f(x)$ for all $x \in N, y \in H$, and such that $f \circ \exp _{\mid \ell} \in \mathscr{S}(k)$, where $k$ is a complement to $h$ in $n$. The topology of $\mathscr{S}\left(N / H, \chi_{\lambda}\right)$ is defined by requiring that the mapping $f \rightarrow f \circ \exp _{\mid \ell}$ be a homeomorphism. Define $P_{\lambda}: \mathscr{S}(N) \rightarrow \mathscr{S}\left(N / H, \chi_{\lambda}\right)$ by

$$
P_{\lambda} f(\exp X)=\int_{h} f(\exp (X+Y)) \chi_{\lambda}(-Y) d Y \text {. }
$$


$P_{\lambda}$ is an open surjection and thus its adjoint $P_{\lambda}^{*}$ is an isomorphism of $\mathscr{S}^{*}\left(N / H, \chi_{\lambda}\right)$ into $\mathscr{S}^{*}(N)$.

Let $h^{\perp}$ be the annihilator of $h$ in $n^{*}$. For $\lambda \in h^{*}$ (identified as a subspace of $\left.n^{*}\right)$, there is a natural Schwartz space on $h^{\perp}+\lambda, \mathscr{S}\left(h^{\perp}+\lambda\right)$, given by composing elements of $\mathscr{S}\left(h^{\perp}\right)$ with translation by $-\lambda$. Considering $\mathscr{S}\left(N / H, \chi_{\lambda}\right)$ and $\mathscr{S}\left(h^{\perp}+\lambda\right)$ as subspaces of $\mathscr{S}^{*}(N)$ and $\mathscr{S}^{*}\left(n^{*}\right)$ respectively, the Fourier transform is defined on these spaces and one has that $f \rightarrow \hat{f}$ is an isomorphism of $\mathscr{S}\left(N / H, \chi_{\lambda}\right)$ onto $\mathscr{S}\left(h^{\perp}+\lambda\right)$ and of $\mathscr{S}\left(h^{\perp}+\lambda\right)$ onto $\mathscr{S}\left(N / H, \chi_{-\lambda}\right)$. Also one has that for $D \in \mathscr{S}^{*}\left(N / H, \chi_{\lambda}\right),\left(P_{\lambda}^{*} D\right)^{\wedge}=R_{-\lambda}^{*} \widetilde{D}$, where $R_{\lambda}: \mathscr{S}\left(n^{*}\right) \rightarrow \mathscr{S}\left(h^{\perp}+\lambda\right)$ is restriction, and $\widetilde{D}$ is the element in $\mathscr{S}^{*}\left(h^{\perp}-\lambda\right)$ defined by $\langle\widetilde{D}, f\rangle=\langle D, \hat{f}\rangle$. Thus $\left(P_{\lambda}^{*} D\right)^{\wedge}$ is supported on $h^{\perp}+\lambda$ and has no normal derivatives.

For $f \in \mathscr{S}\left(N / H, \chi_{\lambda}\right)$ and $D \in \mathscr{S}^{*}\left(N / H, \chi_{-\lambda}\right)$, the convolution $D * f$ is defined by setting $D * f(x)=\left\langle D, l_{x}(\check{f})\right\rangle$ for each $x \in N$. Suppose now that $D \in \mathscr{S}^{*}(N)$ and $f \in \mathscr{S}(N)$. One can use Abelian Fourier analysis to study the mapping defined on $z$, the center of $n$, by $Y \rightarrow D * f(\exp (X+Y))$. If this mapping is in $\mathscr{S}(z)$, then

$$
D * f(\exp X)=\int_{\varkappa^{*}} P_{\lambda}(D * f)(\exp X) d \lambda
$$

for appropriately normalized Lebesgue measure $d \lambda$. Furthermore, $P_{\lambda}(D * f)=$ $D_{\lambda} * P_{\lambda} f$, where $D_{\lambda}$ is the element of $\mathscr{S}^{*}\left(N / H, \chi_{-\lambda}\right)$ whose Fourier transform, $\widetilde{D}_{\lambda}$, agrees with the restriction to $h^{\perp}+\lambda$ of $\widehat{D}$. Thus, convolution between elements of $\mathscr{S}^{*}(N)$ and $\mathscr{S}(N)$ decomposes into convolutions between elements of $\mathscr{S}^{*}\left(N / H, \chi_{-\lambda}\right)$ and $\mathscr{S}\left(N / H, \chi_{\lambda}\right)$ in such a way that smoothness and growth conditions on $\widehat{D}, D \in \mathscr{S}^{*}(N)$ are inherited by $\widetilde{D}_{\lambda}$, $D_{\lambda} \in \mathscr{S}^{*}\left(N / H, \chi_{-\lambda}\right)$. One then proceeds by induction on the dimension of $N / H$. Of course, this requires maintaining considerable control of the various seminorm estimates that appear in the decompositions.

The proof of Theorem B follows along the usual induction argument lines with the Plancherel Theorem being used to reduce the dimension.

For Theorem $\mathrm{C}$, one constructs a $\theta$ on $n^{*}$ such that both $\theta$ and $1 / \theta$ are in $P B_{N}^{\infty}\left(n^{*}\right)$, and such that $\sum\|A(\xi)\| \theta(\xi)<\infty$, the sum being over $(\Gamma \backslash N)_{0}^{\wedge}$. One then uses the fact that $\left(D_{1 / \theta} * f\right) *\left(D_{\theta} * g\right)=f * g$ and the Dixmier and Mallivan [DM] factorization to complete the proof.

REMARKS. The fact that $D_{\theta} \in \mathscr{M} \mathscr{S}(N)$ was proved by R. Howe in [H], and indeed, the ideas presented there are the foundation of this work. Theorem $\mathrm{B}$ was proved for the case where $\theta$ is a polynomial by A. Kirillov in $[\mathbf{K}]$. In [CG], L. Corwin and F. Greenleaf proved Theorem $\mathrm{C}$ with the additional assumption that all the representations in general position were induced from a common, normal subgroup. One-sided Schwartz multipliers have been studied by L. Corwin in $[\mathbf{C}]$. 


\section{REFERENCES}

[C] L. Corwin, Tempered distributions on Heisenberg groups whose convolution with Schwartz class functions is Schwartz class, J. Funct. Anal. 44 (1981), 328-347.

[CG] L. Corwin and F. Greenleaf, Solvability of certain left-invariant differential operators by nilmanifold theory, Comm. Pure Appl. Math. 36 (1983), 755-765.

[DM] J. Dixmier et P. Mallivan, Factorisations de fonctions et de vecteurs indéfiniment différentiables, Bull. Sci. Math. 102 (1978), 305-330.

[H] R. Howe, On a connection between nilpotent groups and oscillatory integrals associated to singularities, Pacific J. Math. 73 (1977), 329-364.

[K] A. Kirillov, Unitary representations of nilpotent Lie groups, Uspekhi Mat. Nauk. 17 (1962), 57-110.

[R] M. Rais, Solutions elémentaires des operateurs différentiels bi-invariants sur un groupe de Lie nilpoteñt, C. R. Acad. Sci. Paris Sér. A-B 273 (1971), A495-498.

[S] L. Schwartz, Théorie des distributions, tome II, Hermann, Paris, 1959.

Department of MAThematics, The UNIVERSity at Albany, State UNiVerSITY OF NEW YORK, ALBANY, NEW YORK 12222 